\title{
P42 Movement Protein of Beet necrotic yellow vein virus Is Targeted by the Movement Proteins P13 and P15 to Punctate Bodies Associated with Plasmodesmata
}

\author{
M. Erhardt, M. Morant, C. Ritzenthaler, C. Stussi-Garaud, H. Guilley, K. Richards, G. Jonard, \\ S. Bouzoubaa, and D. Gilmer \\ Institut de Biologie Moléculaire des Plantes du CNRS et de l'Université Louis Pasteur, 12 rue du Général \\ Zimmer, 67084 Strasbourg cedex, France \\ Accepted 31 January 2000.
}

\begin{abstract}
Cell-to-cell movement of Beet necrotic yellow vein virus (BNYVV) is driven by a set of three movement proteinsP42, P13, and P15-organized into a triple gene block (TGB) on viral RNA 2. The first TGB protein, P42, has been fused to the green fluorescent protein (GFP) and fusion proteins between $\mathrm{P42}$ and GFP were expressed from a BNYVV RNA 3-based replicon during virus infection. GFP-P42, in which the GFP was fused to the P42 N terminus, could drive viral cell-to-cell movement when the copy of the P42 gene on RNA 2 was disabled but the C-terminal fusion P42-GFP could not. Confocal microscopy of epidermal cells of Chenopodium quinoa near the leading edge of the infection revealed that GFP-P42 localized to punctate bodies apposed to the cell wall whereas free GFP, expressed from the replicon, was distributed uniformly throughout the cytoplasm. The punctate bodies sometimes appeared to traverse the cell wall or to form pairs of disconnected bodies on each side. The punctate bodies co-localized with callose, indicating that they are associated with plasmodesmata-rich regions such as pit fields. Point mutations in P42 that inhibited its ability to drive cell-to-cell movement also inhibited GFP-P42 punctate body formation. GFP-P42 punctate body formation was dependent on expression of P13 and P15 during the infection, indicating that these proteins act together or sequentially to localize P42 to the plasmodesmata.
\end{abstract}

Cell-to-cell movement of many plant viruses involves transit of the viral nucleic acid through plasmodesmata in a process dependent on the intervention of one or more virusencoded movement proteins (MPs). Members of the genera Potexvirus, Carlavirus, and Hordeivirus, as well as some fungus-transmitted viruses, including Beet necrotic yellow vein virus (BNYVV; type member of the genus Benyvirus), possess three MPs that are translated from a set of adjacent homologous genes known as the triple gene block (TGB; Gilmer et al. 1992). The three TGB proteins are often referred to generically as TGBp1, TGBp2, and TGBp3.

TGBs have been divided into two classes (Solovyev et al. 1996). In class 1 TGBs (present in BNYVV, the other fungus-

Corresponding author: David Gilmer; Telephone: (33) 3884172 51; Fax: (33) 3886144 42; E-mail: david.gilmer@ibmp-ulp.u-strasbg.fr transmitted viruses, and the hordeiviruses), the first and third TGB proteins are longer than their counterparts in the class 2 TGBs characteristic of the potex- and carlaviruses. Furthermore, in the class 1 TGB-containing viruses that have been studied, viral RNA, presumably in complex with a viral MP, is the infectious entity that trafficks from cell to cell (Petty and Jackson 1990; Gilmer et al. 1992; Herzog et al. 1998), while in the potexviruses (class 2 TGB), virions are thought to be the form active in cell-to-cell movement (Forster et al. 1992; Baulcombe et al. 1995; Santa Cruz et al. 1998).

The TGB proteins of BNYVV are, in order, P42, P13, and P15 (Fig. 1). P42 (also known as TGBp1) has RNA helicase consensus motifs and in vitro nucleic acid binding activity (Bleykasten et al. 1996) and has been reported to be associated with a membrane-enriched, subcellular fraction from virus-infected Chenopodium quinoa leaves (Niesbach-Klösgen et al. 1990). P13 (TGBp2) has N- and C-terminal hydrophobic domains. The exact subcellular localization of P13 has not been established but it is also associated with a membranous subcellular fraction (Niesbach-Klösgen et al. 1990). Finally, P15 (TGBp3) is predicted to be rather hydrophobic in nature but has not been immuno-detected in planta.

The TGB proteins of BNYVV are encoded by RNA 2, which also encodes the viral capsid proteins and a regulatory protein, P14 (Bouzoubaa et al. 1986; Hehn et al. 1995). This RNA and RNA 1, which encodes the viral RNA-dependent RNA replicase (Bouzoubaa et al. 1987), are necessary and sufficient for infection of leaves. In addition to RNAs 1 and 2, field isolates of BNYVV contain two or three smaller RNAs that intervene in the natural infection process (Richards and Tamada 1992). These RNAs are not required for leaf infection but multiply efficiently when coinoculated to leaves with RNAs 1 and 2. We have shown previously that the central region of one of these small RNAs, RNA 3, can be replaced with a foreign gene without interfering with its replication, making it possible to use RNA 3 as a virus replicationdependent expression vector or "replicon" (BleykastenGrosshans et al. 1997; Lauber et al. 1998).

In this paper, we have used the aforesaid replicon to express P42 fused to the green fluorescent protein (GFP) during BNYVV infection of $C$. quinoa leaves. The chimera with GFP fused to the $\mathrm{N}$ terminus of P42 (GFP-P42) could transcomplement cell-to-cell movement when the TGB1 gene on 
RNA 2 had been disabled by mutation, proving that the P42 moiety retained function in the fusion protein. Confocal laser scanning microscopy localized the GFP-P42 to punctate bodies apposed to the cell wall (CW) of infected epidermal cells. The punctate bodies co-localized with callose, a marker for plasmodesmata (Currier and Strugger 1955; Radford et al. 1998; Ritzenthaler et al., in press), suggesting that the bodies are associated with regions with high plasmodesmal density, i.e., pit fields. The GFP-P42 punctate bodies only formed when both of the other two TGB proteins were also expressed.

\section{RESULTS}

A GFP-P42 fusion protein can replace $\mathbf{P 4 2}$

in driving cell-to-cell movement of BNYVV.

The GFP cistron, modified to enhance its expression in eukaryotes (Reichel et al. 1996), was fused in frame to either the $5^{\prime}$ terminus or the $3^{\prime}$ terminus of the P42 cistron. Each gene fusion was then inserted into a plasmid containing the BNYVV RNA 3-based replicon Rep0 (Bleykasten-Grosshans et al. 1997) appropriately positioned to produce RepGFP-P42 and RepP42-GFP (Fig. 1) by run-off transcription. A repliconcontaining plasmid was also constructed for expression of unfused GFP (RepGFP; Fig. 1). The in vitro runoff transcripts were tested for their capacity to multiply following mechanical inoculation to $C$. quinoa leaves along with transcripts corresponding to wild-type BNYVV RNAs 1 and 2. The inoculated leaves developed pale yellow local lesions 4 to 6 days post inoculation. Northern (RNA) hybridization of RNA extracted from the lesions with viral RNA-specific riboprobes established that the three replicons had multiplied (Fig. 2A, lanes 3-5), although the replicons containing GFP fused to either the $\mathrm{N}$ or $\mathrm{C}$ terminus of P42 always accumulated to somewhat lower levels than did the smaller RepGFP. Total proteins were also extracted from the local lesions and tested for the presence of P42-related products by Western blot (immunoblot) with a P42-specific antiserum. In addition to the P42 produced from RNA 2, the protein extracts from the plants inoculated with RepGFP-P42 and RepP42-GFP also contained a polypeptide of approximately $70 \mathrm{kDa}$, close to the size predicted for the GFP-P42 and P42-GFP fusion proteins (Fig. 2B, lanes 4 and 5).

We have shown previously that a replicon containing the gene for P42 (Rep-P42) can trans-complement cell-to-cell movement of BNYVV when the copy of the P42 gene in RNA 2 has been disabled by mutation (Bleykasten-Grosshans et al. 1997). To determine whether the fusion proteins can similarly complement cell-to-cell movement in trans, RepGFP-P42 and RepP42-GFP were each inoculated to $C$. quinoa leaves along with transcripts of wild-type RNA 1 and the RNA 2 mutant, 2$\triangle \mathrm{SB}$, which contains a 405-nucleotide deletion in the $5^{\prime}$ proximal part of the gene for P42 (Fig. 1). Local lesion symptoms were observed on leaves where the RNA 1 plus 2$\Delta \mathrm{SB}$ inoculum had been supplemented with RepGFP-P42 but not when the inoculum was supplemented with RepGFP or RepP42-GFP (data not shown). Progeny viral RNA was detected by Northern blot of the RNA extracted from the leaves when RepGFP-P42 was included in the inoculum (Fig. 2A, lane 10) but not from the leaves inoculated with RNA 1 plus 2- $\triangle \mathrm{SB}$ alone (Fig. 2A, lane 6) or supplemented with RepP42GFP (Fig. 2A, lane 9) or RepGFP (Fig. 2A, lane 7). Western blot analysis revealed that the local lesions resulting from inoculation with the transcripts of RNA 1, 2- $\Delta$ SB, and RepGFPP42 contained only the 70-kDa GFP-P42 fusion protein (Fig. 2B, lane 10), i.e., deletion of the GFP coding region from the replicon did not detectably occur in the course of the experiment. We conclude that fusion of GFP to its $\mathrm{N}$ terminus does not interfere with the ability of $\mathrm{P} 42$ to direct cell-to-cell movement. Fusion of the GFP to the $\mathrm{C}$ terminus of P42, on the other hand, abolished the cell-to-cell movement function of P42.

\section{Subcellular localization of GFP-P42 \\ in leaf epidermal cells.}

C. quinoa leaves were inoculated with BNYVV RNAs 1 and 2 plus RepGFP, RepGFP-P42, or RepP42-GFP and the leaves

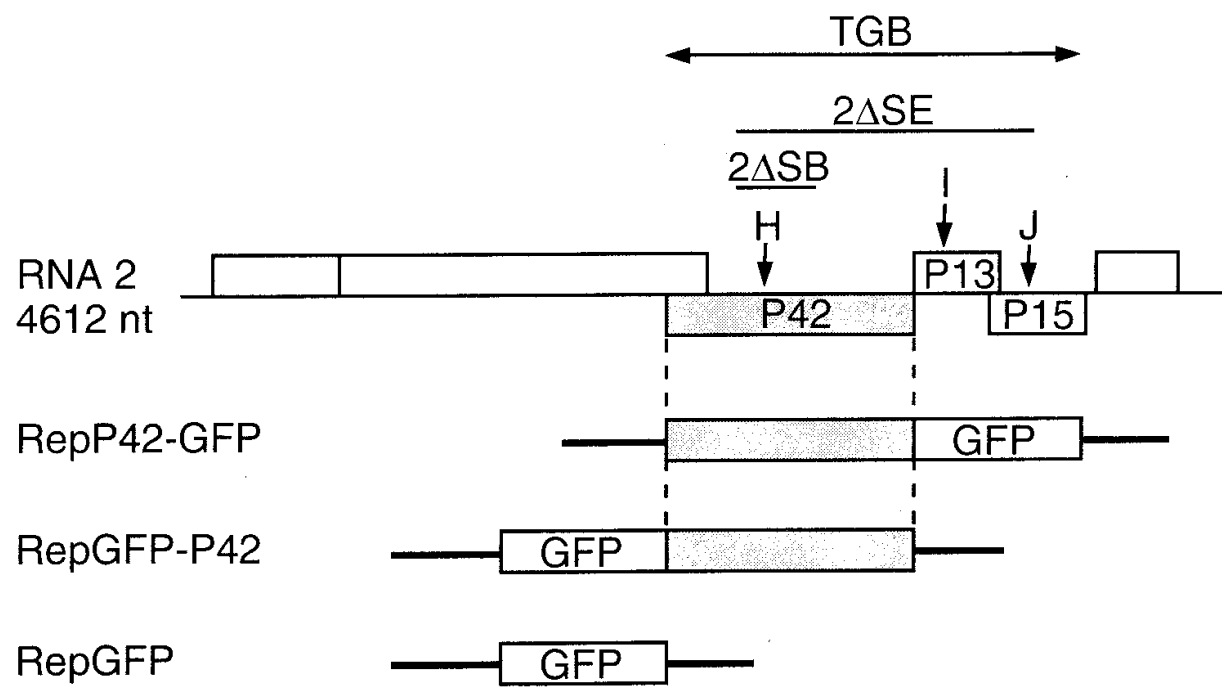

Fig. 1. Map of Beet necrotic yellow vein virus (BNYVV) RNA 2 showing triple gene block (TGB) and BNYVV RNA 3-based replicons containing the gene for the green fluorescent protein (GFP) and P42/GFP gene fusions. Positions of frameshift mutations and deletions in the different TGB proteins mentioned in text are shown above; structures of RepP42-GFP, RepGFP-P42, and RepGFP are shown below. Heavy horizontal lines in the replicons correspond to sequence derived from BNYVV RNA 3. 
were observed by epifluorescence microscopy at different times after inoculation. With all three replicons, small fluorescent disks appeared by 3 to 4 days post inoculation at positions corresponding to sites where lesions were becoming visible in natural light. When RepGFP-P42 or RepP42-GFP were included in the inoculum, the disks subsequently developed into fluorescent rings that expanded with time, with the leading edge of the ring slightly in advance of the visible lesion. When RepGFP was included in the inoculum, the fluorescent disks expanded with time but did not develop into rings during the course of the experiment. These observations suggest that, unlike free GFP, GFP-P42 and P42-GFP are preferentially degraded or cease to be synthesized in the initially infected cells as the infection progresses. Formation of similar expanding fluorescent rings has been reported for plants infected with tobamoviruses expressing MP-GFP fusions (Padgett et al. 1996; Szécsi et al. 1999).

Infected epidermal cells near the periphery of the local lesions were examined 5 to 7 days post inoculation by laser scanning confocal microscopy. When RepGFP was included in the in- oculum, fluorescence was distributed in a uniform manner throughout the cytoplasm of such cells (Fig. 3A) and was also present in the nucleus but no fluorescence was observed within the large vacuoles of the epidermal cells. When the RNA 1 plus RNA 2 inoculum was supplemented with RepP42-GFP (Fig. 3B) or RepGFP-P42 (Fig. 3C), there was a low background of uniform fluorescence in the cytoplasm but, more strikingly, both the GFP-P42- and the P42-GFP-infected cells contained numerous fluorescent punctate bodies apposed to the $\mathrm{CW}$. The bodies were generally 2 to $5 \mu \mathrm{m}$ in diameter.

The punctate bodies were also present in epidermal cells following infection with transcripts of RNA 1, 2- $\Delta \mathrm{SB}$, and RepGFP-P42 (Fig. 3D and E), establishing that the concurrent presence of wild-type P42, derived from full-length RNA 2, is not required for formation of the GFP-P42 punctate bodies. Sometimes, the fluorescent punctate bodies were elongated and appeared to span the $\mathrm{CW}$, while in other cases they formed discrete pairs, one on each side of the wall (see enlargements in Figure 3D and E).

\section{A}
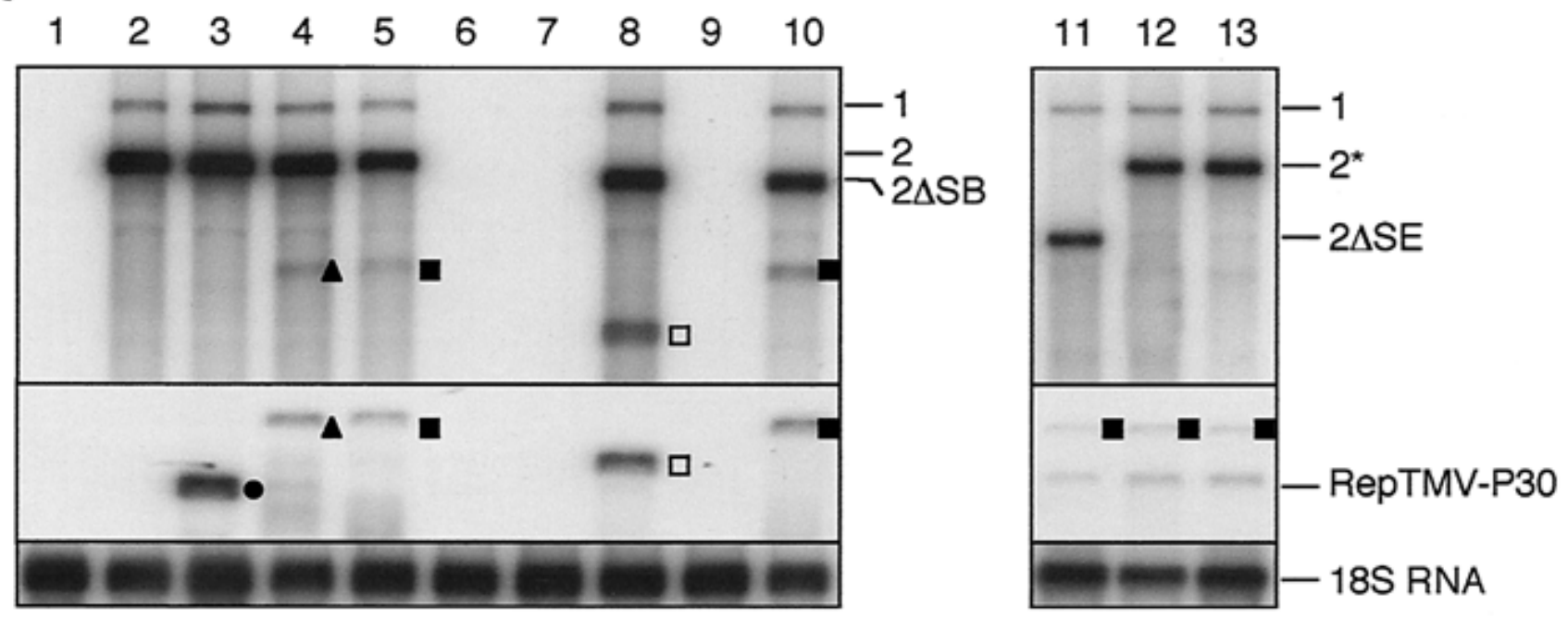

B
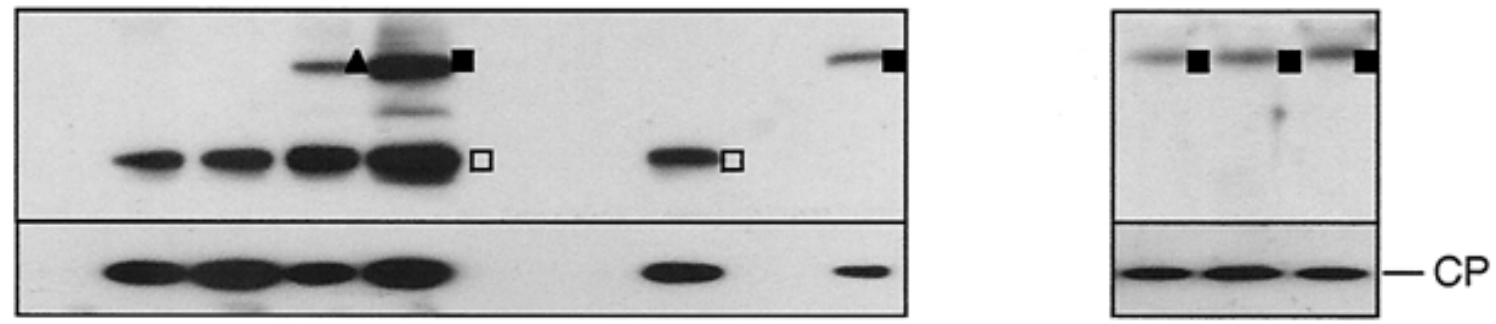

Fig. 2. Detection of viral RNA and protein in leaves infected with Beet necrotic yellow vein virus (BNYVV) RNA 1 and 2 transcripts plus BNYVV RNA 3-based replicons encoding either free green fluorescent protein (GFP), GFP-P42, or P42-GFP. A, Northern (RNA) hybridization to detect BNYVVrelated viral RNAs in total RNA extracted from leaves inoculated with water (lane 1), with wild-type BNYVV RNA 1 and 2 alone (lane 2), or supplemented with RepGFP (lane 3), RepP42-GFP (lane 4), or RepGFP-P42 (lane 5); RNA from leaves inoculated with RNA 1 and 2- $\Delta$ SB alone (lane 6), or supplemented with RepGFP (lane 7), RepP42 (lane 8), RepP42-GFP (lane 9), or RepGFP-P42 (lane 10); RNA from leaves inoculated with RNA 1, RepTMV-P30, and RepGFP-P42 plus 2- $\Delta$ SE (lane 11), 2HI (lane 12), or 2HJ (lane 13). Upper panel hybridized with a mixture of riboprobes specific for RNA 1 and 2 (both probes pB21 and pBS2Sph/Bgl); middle panel with a probe specific for RNA 3; lower panel with a probe specific for 18S ribosomal RNA. RNA species are identified to right or on blot. Bands denoted as $2 *$ correspond to frameshift mutants $2 \mathrm{HI}$ and $2 \mathrm{HJ}$. Bands labeled on blot correspond to RepGFP (filled circle), RepP42-GFP (filled triangles), RepGFP-P42 (filled squares), and RepP42 (hollow squares). B, Total protein extracts from plants inoculated as in A were separated by sodium dodecyl sulfate-polyacrylamide gel electrophoresis, transferred to nitrocellulose, and immunoprobed with a P42-specific antiserum (upper panel) or a viral coat protein-specific antiserum (lower panel). Proteins were identified with reference to mobility markers (not shown) and are labeled on blot or to right: P42-GFP (filled triangles), GFP-P42 (filled squares), and P42 (hollow squares). 


\section{P13 and P15 are required for formation of the GFP-P42} punctate bodies.

Further experiments were carried out to determine whether expression of the second and third TGB proteins, $\mathrm{P} 13$ and P15, is necessary for formation of the GFP-P42 punctate bodies. C. quinoa leaves were inoculated with a mixture of wild-type RNA 1 transcript, an RNA 2 transcript with a deletion eliminating all three TGB proteins (2- $\Delta \mathrm{SE}$; Fig. 1), RepGFP-P42, and RepTMV-P30, a BNYVV RNA 3-based replicon that expresses the Tobacco mosaic virus
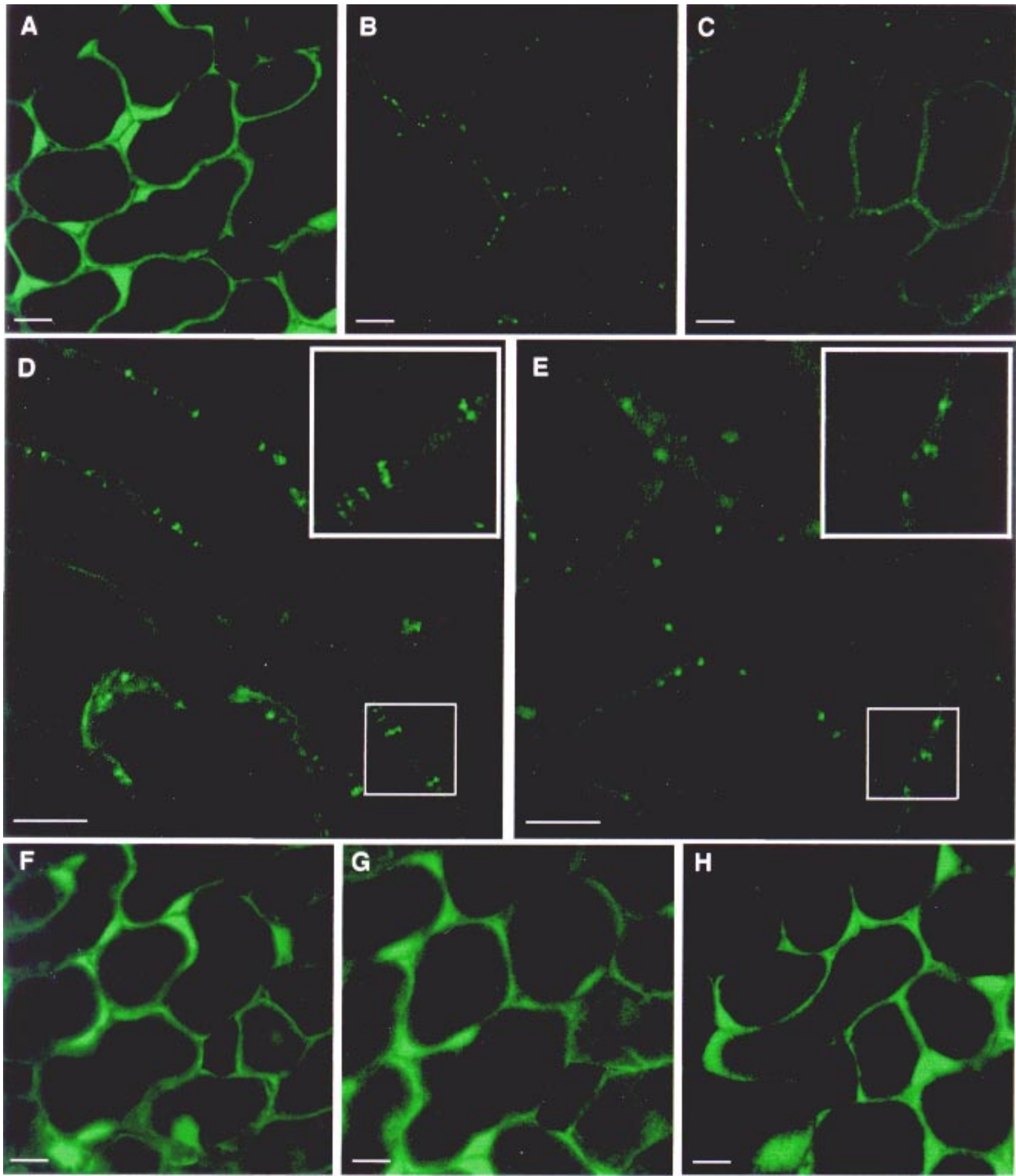

Fig. 3. Visualization by laser scanning confocal microscopy of green fluorescent protein (GFP) fluorescence in epidermal cells near the periphery of local lesions produced by infection with Beet necrotic yellow vein virus (BNYVV) RNA 1 and 2 plus (A) RepGFP, (B) RepP42-GFP, or (C) RepGFP-P42; infection with BNYVV RNA 1 and 2- $\Delta$ SB plus RepGFP-P42 (D and E; inserts at upper right of each panel correspond to a two times magnification of indicated region); infection with RNA 1, RepTMV-P30 and RepGFP-P42 plus (F) RNA 2 mutant 2- $\Delta$ SE, (G) $2 \mathrm{HI}$, or (H) $2 \mathrm{HJ}$. Bars correspond to $50 \mu \mathrm{m}$. 
(TMV) MP P30. We have shown previously that P30 provided in trans can drive BNYVV cell-to-cell movement when one or more of the BNYVV TGB proteins has been disabled by mutation (Lauber et al. 1998). The distribution of GFP-P42 in epidermal cells at the periphery of the local lesions on leaves infected with the aforesaid transcript mix was similar to that observed when free GFP was expressed from the replicon, i.e., the fluorescence was evenly distributed throughout the cytoplasm and nucleoplasm and no punctate bodies were visible (Fig. 3F). A similar uniform distribution of GFP-P42 fluorescence and absence of fluorescent punctate bodies was observed when the genes for P13 and P15 were knocked out separately in the RNA 2 mutants 2HI (Gilmer et al. 1992), which contains frameshift mutations in the P42 and P13 cistrons, and 2HJ (Gilmer et al. 1992), which contains frameshift mutations in the $P 42$ and P15 cistrons (Fig. 3G and H). We conclude that both $\mathrm{P} 13$ and $\mathrm{P} 15$ are required for targeting of P42 to the punctate bodies.
Effects of mutations in P42 on punctate body formation.

P42 and its homologues in the other TGB-containing viruses contain a set of sequence motifs resembling the RNA helicase consensus motifs present in known or putative viral replicases (Gorbalenya and Koonin 1989). This suggests that the TGBp1 proteins may be able to unfold RNA secondary structure. Point mutations, generally substitution of alanine (A) for conserved and/or charged residues, were created at various sites in P42 including certain of the helicase consensus motifs (Fig. 4). The genes encoding the mutant forms of P42 were introduced into the RNA 3 replicon and the replicons were then tested for their ability to drive cell-to-cell movement when coinoculated to $C$. quinoa with RNA 1 plus $2-\Delta \mathrm{SB}$ transcripts as before. Of the mutants targeting the helicase consensus motifs I, II, and V, only mutant Ala10, which targeted motif $\mathrm{V}$, retained the ability to transcomplement movement (Fig. 4), as evidenced by the appearance of local lesions on the inoculated leaves and progeny viral RNA detectable by Northern blot (data not shown).

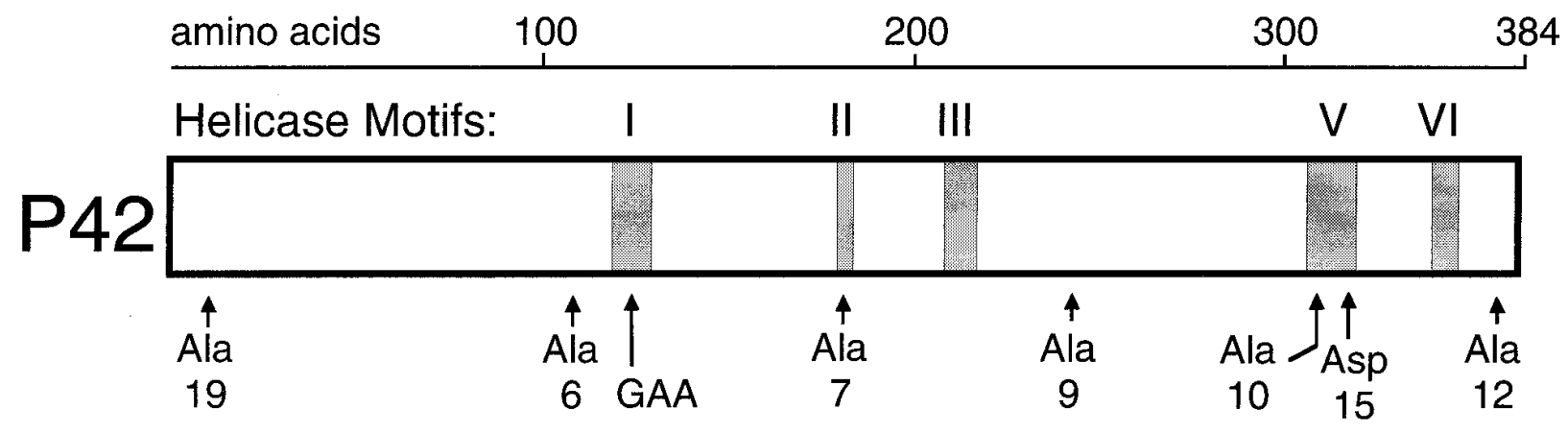

\begin{tabular}{|c|c|c|c|c|}
\hline Mutant & $\begin{array}{l}\text { Wild-type } \\
\text { Sequence }\end{array}$ & $\begin{array}{c}\text { Mutant } \\
\text { Sequence }\end{array}$ & $\begin{array}{l}\text { Cell-to-cell } \\
\text { Movement }\end{array}$ & $\begin{array}{l}\text { Punctate } \\
\text { Formatic }\end{array}$ \\
\hline Ala19 & $\underline{\mathrm{RRTGGDKGAK}} \underline{\mathrm{K}}(6-15)$ & AATGGDAGAA & + & NT \\
\hline Ala6 & $\underline{\operatorname{KVKT}}(107-110)$ & AVAA & + & + \\
\hline GAA & $\mathrm{GKS}(128-130)$ & GAA & - & - \\
\hline Ala7 & $\operatorname{VDE}(187-189)$ & VAA & - & - \\
\hline Ala9 & $\underline{\mathrm{RRFGK}} \underline{\mathrm{K}}(242-246)$ & AAFGA & - & - \\
\hline Ala10 & DAHGQ(310-314) & AAAGA & + & + \\
\hline Asp15 & VTIIL(318-322) & VTDDD & - & - \\
\hline Ala12 & $\operatorname{KNGD}(362-365)$ & AAGA & - & NT \\
\hline
\end{tabular}

Fig. 4. Positions of point mutations in P42 and their effects on cell-to-cell movement and punctate body formation. Map shows positions of mutations and helicase consensus motifs I, II, III, V, and VI (no sequence corresponding to the relatively poorly conserved motif IV could be identified). For each mutation, wild-type and mutant sequences are indicated in the table below with the amino acid alterations underlined. Numbers in parentheses to the right: amino acid coordinates in P42. RNA 3-based replicons carrying each mutant P42 were tested for their ability to support viral cell-to-cell movement (lesion formation and progeny viral RNA detectable by Northern [RNA] hybridization) following inoculation of Chenopodium quinoa with the replicon plus RNA 1 and 2- $\Delta$ SB. Mutations were also introduced into the P42 sequence of RepGFP-P42 and the ability of resulting mutant GFP-P42 fusion proteins to form punctate bodies in epidermal cells on periphery of local lesions following inoculation of $C$. quinoa with the replicon plus RNAs 1 and 2 was examined. $\mathrm{NT}=$ not tested. 
Mutations outside of the helicase consensus motifs could also affect cell-to-cell movement. Thus, mutant Ala12, in which charged residues downstream of motif VI were replaced by alanines, was movement-defective as was Ala9, in which the three charged residues in the sequence ${ }_{242} \mathrm{RRFGK}_{246}$ were replaced by As. Mutants Ala6 and Ala19, on the other hand, in which the modifications map to the region upstream of the helicase consensus motifs, were still capable of transcomplementing cell-to-cell movement (Fig. 4).

To determine what effect point mutations in $\mathrm{P} 42$ have on punctate body formation, the mutations GAA, Ala7, Ala9, and Asp15 (all of which abolish movement), and Ala6 and Ala10 (both functional in cell-to-cell movement) were engineered into RepGFP-P42 and the resulting replicons were inoculated to $C$. quinoa with wild-type BNYVV RNA 1 and 2. All of the mutants gave rise to fluorescence in the resulting local lesions but only the nonlethal mutants Ala6 and Ala10 produced punctate bodies in epidermal cells similar to those observed with wild-type GFP-P42 (Fig. 4). Thus, so far, we have been unable by point mutation to uncouple the activity of P42 in movement from its ability to form punctate bodies.

\section{The $\mathbf{P 4 2}$ punctate bodies are localized to regions of the $\mathrm{CW}$ rich in plasmodesmata.}

We have reported previously that P42 is primarily present in a membrane-enriched subcellular pellet (produced by centrifugation at $30,000 \times g)($ Niesbach-Klösgen et al. 1990) isolated from BNYVV-infected $C$. quinoa leaves, although more recent experiments have detected significant amounts of $\mathrm{P} 42$ in a $\mathrm{CW}$-enriched fraction as well (M. Erhardt, unpublished observations). To obtain additional information about the subcellular localization of $\mathrm{P} 42$, a fraction enriched in CW fragments was prepared from $C$. quinoa leaves infected with an inoculum consisting of transcripts of RNA 1, 2- $\Delta \mathrm{SB}$, and RepGFP-P42. Before observation, the material in the CW fraction was treated with a mouse monoclonal antibody specific for callose, a marker for plasmodesmata (Northcote et al. 1989; Benhamou 1992; Turner et al. 1994), followed by treatment with sheep anti-mouse Alexa 546-labeled antibodies to immuno-label the callose with a distinct fluorescent marker. The CW fraction was then examined by differential interference contrast microscopy to identify structures (Fig. 5A, E, and I) and by epifluorescence microscopy with different filter sets to differentiate between the fluorescence due to GFP (Fig. $5 \mathrm{~B}, \mathrm{~F}$, and $\mathrm{J}$ ) and that due to the Alexa 546 reagent (Fig. 5C, $\mathrm{G}$, and $\mathrm{K}$ ).

Green fluorescent punctate bodies like those visualized by laser scanning confocal microscopy of intact cells were frequently observed in association with the $\mathrm{CW}$ fragments. The similarity of these structures to the fluorescent punctate bodies observed by confocal microscopy of intact cells was particularly apparent where the $\mathrm{CW}$ fragment was folded so that portions of it were positioned parallel to the line of view (see Figure 5A and B). Examination of the same regions for Alexa 546-fluorescence revealed that the callose-specific marker frequently co-localized with the GFP-punctate bodies (Fig. 5D and $\mathrm{H}$ ). Such co-localization was particularly evident when $\mathrm{CW}$ fragments were flattened against the slide so that they could be seen in overhead view (Fig. 5H). No fluorescent punctate bodies were observed in association with the CW fragments when free GFP was expressed from the replicon
(Fig. 5J). We conclude that most if not all of the GFP-P42 punctate bodies are apposed to regions of the $\mathrm{CW}$ rich in plasmodesmata, probably pit fields.

\section{DISCUSSION}

In this paper we have used a BNYVV RNA 3-based replicon to express a GFP-P42 fusion protein in infected plants. RepGFP-P42 is dependent on BNYVV RNAs 1 and 2 for its replication and for cell-to-cell movement but the GFP-P42 produced from the replicon can in turn drive viral cell-to-cell movement when the copy of the P42 gene present on BNYVV RNA 2 has been disabled by mutation. Since fusion of the GFP moiety to the $\mathrm{N}$ terminus of P42 did not interfere with its function in movement, it seems reasonable to conclude that the in situ localization of GFP-P42 reflects that of wild-type P42.

Fusion of GFP to the $\mathrm{C}$ terminus of P42, on the other hand, abolished its ability to support cell-to-cell movement. There is circumstantial evidence that the C-terminal half of $\mathrm{P} 42$, where the consensus helicase motifs reside, represents a "core" functional domain of the protein since the shorter TGBp1s of the potex- and carlaviruses contain only the helicase consensus region and can still drive cell-to-cell movement. Furthermore, for the potexvirus Potato virus $X$ (PVX), the region of TGBp1 involved in interaction with other viral proteins has been mapped to the C-terminal part of the helicase domain (Morozov et al. 1999). If P42 enjoys a similar functional topology, addition of the bulky P42 moiety just downstream of the helicase consensus region could interfere with binding of P42-GFP to other components of the movement complex, possibly including other P42 molecules. Interestingly enough, and in contrast to the GFP-P42 mutants containing lethal point mutations (which presumably interfere in a dominant manner with targeting of the fusion protein to punctate bodies), P42GFP localized to $\mathrm{CW}$-associated punctate bodies. It should be kept in mind, however, that, in the subcellular localization experiments with P42-GFP, P42 produced from the wild-type RNA 2 present in the inoculum can also participate in punctate body formation and the hypothetical presence in the bodies of both P42 and P42-GFP could relieve packing problems that might occur if only P42-GFP were present. In several experiments, RepP42-GFP was coinoculated to $C$. quinoa leaves with RNA 1 and 2- $\Delta$ SB transcript (P42 gene partially deleted), and the inoculum was supplemented with RepTMV-P30 to provide movement functions. However, for unknown reasons, the RepP42-GFP was rapidly lost in the course of the infection and no fluorescence could be detected near the periphery of the lesions. Thus, whether P42-GFP can form punctate bodies in the absence of wild-type $\mathrm{P} 42$ remains an open question.

Not unexpectedly, point mutations in the conserved helicase consensus motifs of P42 generally abolished cell-to-cell movement. The exception to this rule was mutant Ala10, which targeted motif V (Fig. 4). It should be noted, however, that the wild-type motif $\mathrm{V}$ sequence in P42, LYSDA $\underline{H}$ GQTYSVVTII (residues replaced by A in mutant Ala10 underlined), does not correspond perfectly to the viral replicase

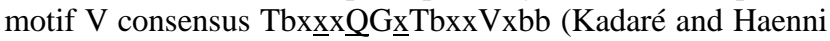
1997; underlined residues correspond to positions where an A was introduced in mutant Ala10; $\mathrm{b}$ is a bulky residue and $\mathrm{x}$ is any residue), or to the very similar motif $\mathrm{V}$ consensus based 
on comparisons of other Class 1 TGBs (not shown). Furthermore, of the three residues targeted for mutation in Ala10, only the $\mathrm{H}$ to A substitution altered a residue at a conserved position in the consensus sequence and the $\mathrm{H}$ present in the wild-type P42 sequence does not correspond to the residue (Q) present at this position in the replicase or TGB consensus sequences. In mutant Asp15, on the other hand, the two conserved bulky residues in the penultimate and last positions of motif $\mathrm{V}$ were replaced by Ds and this alteration inhibited cellto-cell movement (Fig. 4). Thus, the failure of the Ala10 mutation to inhibit cell-to-cell movement does not rule out a potential role for a $\mathrm{P} 42$ helicase activity in the process.

Mutational analysis of the PVX TGBp1 has implicated the region containing helicase motifs I and II in in vitro nucleic acid binding (Morozov et al. 1999). Earlier studies with P42, on the other hand, indicated that this region was dispensable for in vitro nucleic acid binding but that the N-terminal 24 amino acids of the protein were important (Bleykasten et al. 1996). The N-terminal sequence in question contains three $R$ and two $\mathrm{K}$ residues that might possibly interact with the nucleic acid. Four of these five residues were replaced by As in mutant Ala19 but, surprisingly, the mutant was still functional in cell-to-cell movement (Fig. 4). We conclude that either (i) the basic residues targeted in Ala19 are not involved in nucleic acid binding, or (ii) the nucleic acid binding activity observed in vitro is not related to in vivo P42 function. More generally speaking, these observations indicate the need for caution in relating in vitro nucleic acid binding activity of MPs to the situation in vivo.

The parietal localization of the GFP-P42 punctate bodies is evidently consistent with the known role for the TGB proteins and more particularly TGBp1 in plasmodesmal gating (Angell et al. 1996; Lough et al. 1998). It is also consistent with our previous localization by immunogold electron microscopy of the analogous Peanut clump virus TGBp1 protein (P51) to the collar region of plasmodesmata (Erhardt et al. 1999), although the electron microscopic observations of the anti-P51 labeled plasmodesmata provided no evidence for morphologically distinct structures that might correspond to the GFP-P42 punctate bodies described in this paper. In the earlier study, we showed that P51 expressed constitutively in transgenic plants only localized to the plasmodesmata when the plants
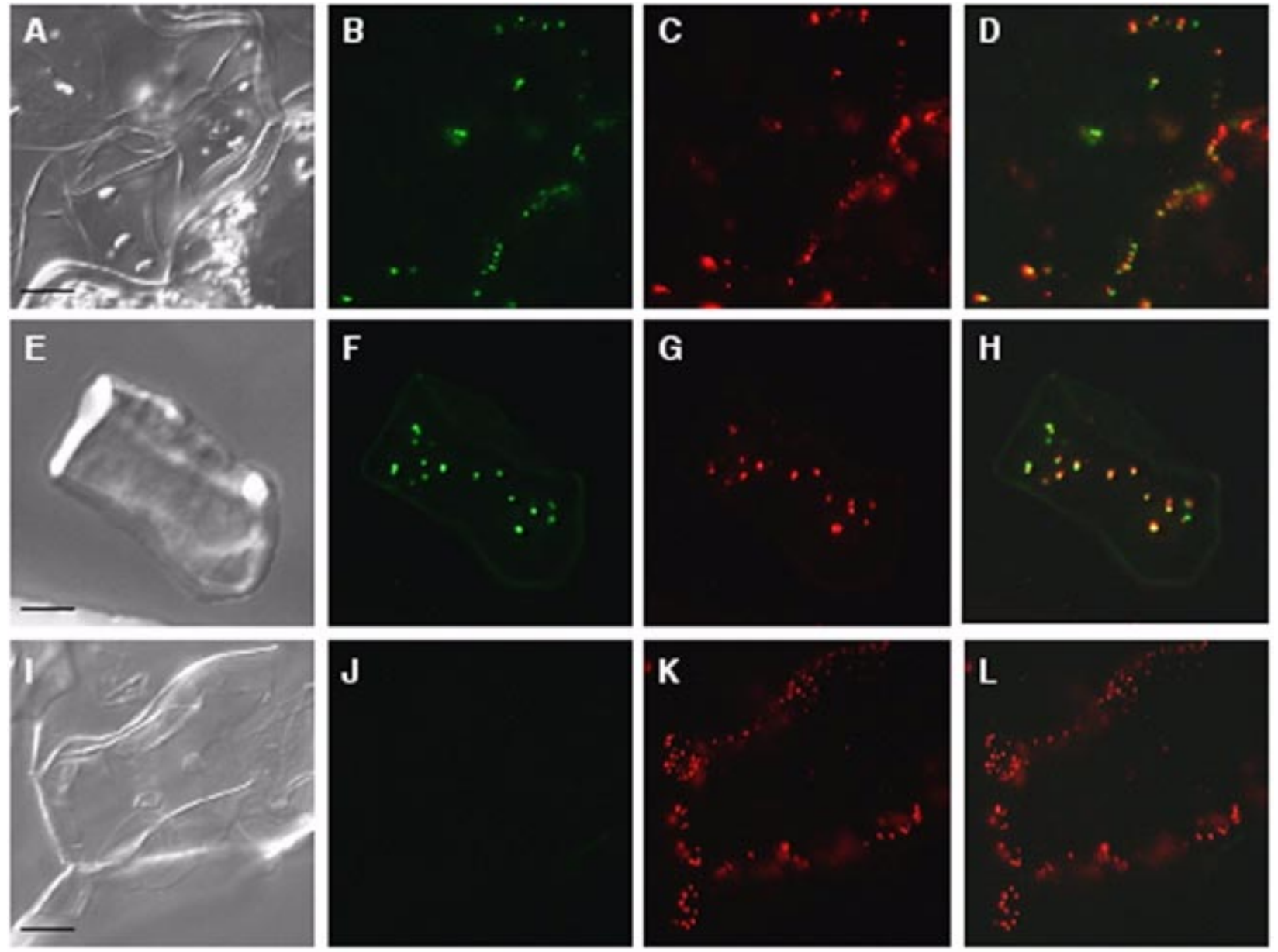

Fig. 5. GFP-P42 and callose co-localize in cell wall $(\mathrm{CW})$ fragments. Chenopodium quinoa leaves were infected with (A-H) RNA $1,2-\Delta \mathrm{SB}$, and RepGFP-P42 or (I-L) RNA 1, RNA 2, and RepGFP. CW fragments were prepared 6 days post infection and material was examined by (A, E, and I) differential interference contrast microscopy to visualize the overall morphology of fragments. A-D, Sample is a CW fragment in which a portion is aligned perpendicular to line of view by contact with a second fragment to the right; $\mathbf{E}-\mathbf{H}$ and $\mathbf{I}-\mathbf{L}$, Samples are CW fragments in transverse view. B, F, and $\mathbf{J}$, Observations of GFP-P42 fluorescence. $\mathbf{C}, \mathbf{G}$, and $\mathbf{K}$, Observations of callose (after reaction with a callose-specific antibody followed by immunolabeling with fluorescent marker Alexa 546). D, H, and L, Superposition of GFP- and callose-specific fluorescence. Bars correspond to $50 \mu \mathrm{m}$. 
were virus infected (Erhardt et al. 1999). The observations in this paper have identified the two other TGB proteins P13 and P15 as essential for P42 punctate body formation but do not rule out the possibility that other viral proteins as well as cellular proteins may also be required.

The fact that the GFP-P42 punctate bodies sometimes appear to traverse the $\mathrm{CW}$ or to form apposing pairs suggests that some if not all of the bodies are located at sites on the $\mathrm{CW}$ where there is extensive cytoplasmic connectivity between the adjacent cells, i.e., areas rich in plasmodesmata such as pit fields. This line of reasoning is reinforced by the observation that the punctate bodies co-localize with callose-rich regions on $\mathrm{CW}$ fragments, since callose is thought to be rapidly deposited in plasmodesmata during the wound response elicited by sample preparation for microscopy (Hughes and Gunning 1980; Radford et al. 1998), with plasmodesmata-rich pit fields representing a particularly strong site for callose deposition (Currier and Strugger 1955; Ritzenthaler et al., in press).

Paired fluorescent punctate bodies similar to those visualized in this study have been observed near the leading edge of lesions on Nicotiana benthamiana inoculated with TMV transcripts expressing TMV P30 tagged with GFP (Oparka et al. 1997; Szécsi et al. 1999). In the case of TMV, the punctate bodies were also shown to contain the viral replicase proteins (Szécsi et al. 1999). The bodies probably correspond to the previously described viroplasms (Saito et al. 1987) and may represent sites where viral RNA replication and cell-to-cell movement are coupled. At present we have no direct evidence for the presence of other BNYVV-encoded proteins in the GFP-P42 punctate bodies. However, the fact that P13 and P15 are required for targeting of $\mathrm{P} 42$ to the bodies suggests strongly that one or both of these proteins are at least transiently present there. Further experiments will be aimed at characterizing the protein composition of the P42-containing punctate bodies in more detail.

\section{MATERIALS AND METHODS}

\section{Clones and infectious transcripts.}

Clones and procedures for production of run-off transcripts of RNA 1, RNA 2, 2- $\triangle \mathrm{ES}$, and RepTMV-P30 have been described (Quillet et al. 1989; Bleykasten-Grosshans et al. 1997; Lauber et al. 1998). The P42 (Bouzoubaa et al. 1986) deletion mutant $2-\Delta \mathrm{SB}$ was produced by eliminating the sequence between the SpeI and BstXI sites and the TGB deletion mutant $2-\Delta \mathrm{SE}$ was produced by eliminating the sequence between the SpeI and EcoRI sites. Fusions between P42 and GFP (Reichel et al. 1996) were generated by overlap extension mutagenesis (Ho et al. 1989) of cloned cDNA during the polymerase chain reaction (PCR). In RepGFP-P42, the GFP termination codon was replaced by $\mathrm{CCC}$ and the $\mathrm{P} 42$ initiation codon by GGG; in RepP42-GFP, the P42 termination codon was replaced by CCC and the GFP initiation codon by GGG. Point mutations in $\mathrm{P} 42$ were also created by overlap extension mutagenesis.

\section{Infection of leaves and analysis of infection products.}

Transcripts were inoculated to fully expanded leaves of $C$. quinoa (four- to six-leaf stage) as described (Lauber et al. 1998). Viral RNA in infected tissue was detected by Northern hybridization with ${ }^{32} \mathrm{P}$-labeled anti-sense RNA probes. Probes obtained by transcription of pB12 (RNA 1-specific), pB21 RNA 2-specific), and pB34 (RNA 3-specific) have already been described (Lemaire et al. 1988). To obtain a probe specific for the portion of RNA 2 upstream of the TGB, the SphI$B g l$ II fragment (residues 252 to 2080) of RNA 2 was cloned into the SphI-BamHI sites of pBSM (Stratagene, La Jolla, $\mathrm{CA})$. The resulting plasmid $(\mathrm{pBS} 2 \mathrm{Sph} / \mathrm{Bgl})$ was linearized with HindIII and a ${ }^{32} \mathrm{P}$-labeled anti-sense RNA probe was prepared by run-off transcription with bacteriophage T7 RNA polymerase as before (Lemaire et al. 1988).

Polypeptides containing the sequence of P42 or the viral coat protein were immuno-detected on Western (protein) blots with specific antisera (Niesbach-Klösgen et al. 1990) except that immunolabeled bands were visualized with an enhanced chemiluminescence kit (Pierce, Rockford, IL) following the supplier's instructions. To verify that the P42 point mutations were conserved in the progeny RNA following successful infection, a sequence of several hundred nucleotides spanning each mutation was amplified by reverse transcription followed by PCR with appropriate primers. The PCR fragments were then cloned and sequenced.

\section{Detection of GFP and callose.}

GFP fluorescence in epidermal cells of infected $C$. quinoa leaves was visualized by laser scanning confocal microscopy with the $488 \mathrm{~nm}$ ray of either a Zeiss LSM 510 microscope (Argon laser; emission band pass 505 to $530 \mathrm{~nm}$ ) or an Olympus BX50 Fluoview (Argon/Krypton laser; emission band pass 510 to $530 \mathrm{~nm}$ ) microscope. The $\mathrm{CW}$ fraction from virusinfected leaves was prepared as described (Ritzenthaler et al., in press) and the CWs were immobilized on glass slides coated with poly-L-lysine. Callose was immunolabeled with a $1 / 100$ dilution of mouse monoclonal anti- $\beta(1-3)$ glucan antibody (Biosupplies, Parkville, Australia), followed by treatment with Alexa 546 goat anti-mouse IgG conjugate (Molecular Probes, Eugene, OR) as described (Ritzenthaler et al., in press). Callose-specific epifluorescence was detected with a Nikon Eclipse E800 microscope equipped with a filter set giving $\lambda_{\text {exc }}$ of $540 \pm 12.5$, a dichroic mirror at $565 \mathrm{~nm}$, and $\lambda_{\text {em }}$ of $605 \pm 27.5 \mathrm{~nm}$. GFP epifluorescence was detected with a filter set giving $\lambda_{\text {exc }}$ of 460 to $500 \mathrm{~nm}$ and $\lambda_{\mathrm{em}}$ of $510 \mathrm{~nm}$. Images were captured with a Sony DXC 950P camera and digitized with Visiolab 200 software (Biocom, Les Ulis, France). Images were corrected for brightness and contrast and further manipulated with Photoshop (Adobe Systems, Mountain View, CA).

\section{ACKNOWLEDGMENTS}

We thank Esther Lechner and Emmanuelle Lauber for help with some of the constructions, Philippe Hammann for sequence analysis, and Daniele Scheidecker for technical aid. We would also like to thank both Zeiss and Olympus for allowing us to make some of the reported observations with their confocal microscopes during training demonstrations.

\section{LITERATURE CITED}

Angell, S. M., Davies, C., and Baulcombe, D. C. 1996. Cell-to-cell movement of potato virus $\mathrm{X}$ is associated with a change in the sizeexclusion limit of plasmodesmata in trichome cells of Nicotiana clevelandii. Virology 316:197-201.

Baulcombe, D. C., Chapman, S. N., and Santa Cruz, S. 1995. Jellyfish 
green fluorescent protein as a reporter for virus infections. Plant J. 7: 1045-1053.

Benhamou, N. 1992. Ultrastructural detection of $\beta-1,3$-glucans in tobacco root tissues infected by Phytophthora parasitica var. nicotianae using a gold complexed tobacco $\beta-1,3$-glucanase. Physiol. Mol. Plant Pathol. 41:351-370.

Bleykasten, C., Gilmer, D., Guilley, H., Richards, K. E., and Jonard, G. 1996. The beet necrotic yellow vein virus $42 \mathrm{kDa}$ triple gene block protein binds nucleic acids in vitro. J. Gen. Virol. 77:889-897.

Bleykasten-Grosshans, C., Guilley, H., Bouzoubaa, S., Richards, K. E., and Jonard, G. 1997. Independent expression of the first two triple gene block proteins of beet necrotic yellow vein virus complements virus defective in the corresponding gene but expression of the third protein inhibits viral cell-to-cell movement. Mol. Plant-Microbe Interact. 10:240- 246.

Bouzoubaa, S., Quillet, L., Guilley, H., Jonard, G., and Richards, K. 1987. Nucleotide sequence of beet necrotic yellow vein virus RNA 1. J. Gen. Virol. 68:615-626.

Bouzoubaa, S., Ziegler, V., Beck, D., Guilley, H., Richards, K., and Jonard, G. 1986. Nucleotide sequence of beet necrotic yellow vein virus RNA 2. J. Gen. Virol. 67:1689- 1700.

Currier, H. B., and Strugger, S. 1955. Aniline blue and fluorescence microscopy of callose in bud scales of Allium cepa L. Protoplasma 45: 552-559.

Erhardt, M. Stussi-Garaud, C., Guilley, H., Richards, K. E., Jonard, G., and Bouzoubaa, S. 1999. The first triple gene block protein of peanut clump virus localizes to the plasmodesmata during virus infection. Virology 264:220-229.

Forster, R. L. S., Beck, D. L., Guilford, P. J., Voot, D. M., and Andersen M. T. 1992. The coat protein of white clover mosaic potexvirus has a role in facilitating cell-to-cell transport in plants. Virology 191:480484.

Gilmer, D., Bouzoubaa, S., Hehn, A., Guilley, H., Richards, K., and Jonard, G. 1992. Efficient cell-to-cell movement of beet necrotic yellow vein virus requires $3^{\prime}$ proximal genes located on RNA 2. Virology 189:40-47.

Gorbalenya, A. E., and Koonin, E. V. 1989. Viral proteins containing the purine NTP-binding sequence pattern. Nucleic Acids Res. 17:84138440.

Hehn, A., Bouzoubaa, S., Bate, A., Twell, D., Marbach, J., Richards, K., Guilley, H., and Jonard, G. 1995. The small cysteine-rich protein P14 of beet necrotic yellow vein virus regulates accumulation of RNA 2 in cis and coat protein in trans. Virology 210:73-81.

Herzog, E., Hemmer, O., Hauser, S., Meyer, G. Bouzoubaa, S., and Fritsch, C. 1998. Identification of genes involved in replication and movement of peanut clump virus. Virology 248:312-322.

Ho, S. N., Hunt, H. D., Horton, R. M., Pullen, J. K., and Pease, L. 1989. Site-directed mutagenesis by overlap extension using the polymerase chain reaction. Gene 77:51-59.

Hughes, J. E., and Gunning, B. E. S. 1980. Gluteraldehyde-induced deposition of callose. Can. J. Bot. 58:250-258.

Kadaré, G., and Haenni, A. L. 1997. Virus-encoded RNA helicases. J. Virol. 71:2583-2590

Lauber, E., Bleykasten-Grosshans, C., Erhardt, M., Bouzoubaa, S., Jonard, G., Richards, K. E., and Guilley, H. 1998. Cell-to-cell movement of beet necrotic yellow vein virus: I. Heterologous complementation experiments provide evidence for specific interactions among the triple gene block proteins. Mol. Plant-Microbe Interact. 11:618-625.

Lemaire, O., Merdinoglu, D., Valentin, P., Putz, C., Ziegler-Graff, V.,
Guilley, H., Jonard, G., and Richards, K. 1988. Effect of beet necrotic yellow vein virus RNA composition on transmission by Polymyxa betae. Virology 162:232-235.

Lough, T. J., Shash, K., Xoconostle-Cázares, B., Hofstra, K. R., Beck, D. L., Balmori, E., Forster, R. L. S., and Lucas, W. J. 1998. Molecular dissection of the mechanism by which potexvirus triple gene block proteins mediate cell-to-cell transport of infectious RNA. Mol. PlantMicrobe Interact. 11:801-814.

Morozov, S. Y., Solovyev, A. G., Kalinina, N. O., Fedorkin, O. N., Samuilova, O. V., Schiemann, J., and Atabekov, J. G. 1999. Evidence for two nonoverlapping functional domains in the potato virus $\mathrm{X} 25 \mathrm{~K}$ movement protein. Virology 260:55-63.

Niesbach-Klösgen, U., Guilley, H., Jonard, G., and Richards, K. 1990. Immunodetection in vivo of beet necrotic yellow vein virus-encoded proteins. Virology 178:52-61.

Northcote, D. H., Davey, R., and Lay, J. 1989. Use of antisera to localize callose, xylan and arabinogalactan in the cell-plate, primary and secondary walls of plant cells. Planta 178:353-366.

Oparka, K. J., Prior, D. A. M., Santa Cruz, S., Padgett, H. S., and Beachy, R. N. 1997. Gating of epidermal plasmodesmata is restricted to the leading edge of expanding infection sites of tobacco mosaic virus (TMV). Plant J. 12:781-789.

Padgett, H. S., Epel, B. L., Kahn, T. W., Heinlein, M., Watanabe, Y., and Beachy, R. 1996. Distribution of tobamovirus movement protein in infected cells and implications for cell-to-cell spread of infection. Plant J. 10:1079-1088.

Petty, I. T. D., and Jackson, A. O. 1990. Mutational analysis of barley stripe mosaic virus RNA $\beta$. Virology 179:712-718.

Quillet, L., Guilley, H., Jonard, G., and Richards, K. 1989. In vitro synthesis of biologically active beet necrotic yellow vein virus RNAs. Virology 172:293-301.

Radford, J. E., Vesk, M., and Overall, R. L. 1998. Callose deposition at plasmodesmata. Protoplasma 201:30-37.

Reichel, C., Mathur, J., Eckes, P., Langenkemper, K., Koncz, C., Schell, J., Reiss, B., and Maas, C. 1996. Enhanced green fluorescence by the expression of an Aequorea victoria green fluorescent protein mutant in mono- and dicotyledonous plant cells. Proc. Natl. Acad. Sci. USA 93:5888-5893.

Richards, K. E., and Tamada, T. 1992. Mapping functions on the multipartite genome of beet necrotic yellow vein virus. Annu. Rev. Phytopathol. 30:291-313.

Ritzenthaler, C., Findlay, K., Roberts, K., and Maule, A. J. Rapid detection of plasmodesmata in purified cell walls. Protoplasma. (In press.)

Saito, T., Hosokawa, D., Meshi, T., and Okada, Y. 1987. Immunocytochemical localization of the $130 \mathrm{~K}$ and $180 \mathrm{~K}$ (putative replicase component) of tobacco mosaic virus. Virology 160:477-481.

Santa Cruz, S., Roberts, A. G., Prior, D. A. M., Chapman, S., and Oparka, K. J. 1998. Cell-to-cell and phloem-mediated transport of potato virus X: The role of virions. Plant Cell 10:495-510.

Solovyev, A. G., Savenkov, E. I., Agranovsky, A. A., and Morozov, S. Y. 1996. Comparisons of the genomic cis-elements and coding regions in RNA $\beta$ components of the hordeiviruses barley stripe mosaic virus, lychnis ringspot virus, and poa semilatent virus. Virology 219: 9-18.

Szécsi, J., Ding, X. S., Lim, C. O., Bendahmane, M., Cho, M. J., Nelson R. S., and Beachy, R. N. 1999. Development of tobacco mosaic virus infection sites in Nicotiana benthamiana. Mol. Plant-Microbe Interact. 12:143-152.

Turner, A., Wells, B., and Roberts, K. 1994. Plasmodesmata of maize root tips: structure and composition. J. Cell Sci. 107:3351-3361. 